\title{
Is Aerated Soft Drink and Packaged Juice Consumption an Independent Risk Factor to Cause Non-Alcoholic Fatty Liver Disease
}

\author{
Kukreja Kunal ${ }^{1}$, Kinra Prateek ${ }^{2 *}$ and Tevatia MS ${ }^{3}$ \\ ${ }^{1}$ Under-graduate, Armed Forces Medical College, Pune, India \\ ${ }^{2}$ Dept of Pathology, Armed Forces Medical College, Pune, India \\ ${ }^{3}$ Dept of Pathology, CH (SC), Pune, India
}

\begin{abstract}
Background: There has been an increase in consumption of aerated sweetened soft drinks and packaged juices high in carbohydrates/fructose by humans across the globe. There have been various studies with contradictory inferences of association of chronic sweetened soft drink (rich in high fructose corn syrup) intake and NAFLD. This study was undertaken with the aim to determine the quantity, frequency, duration and type of sweet soft drinks /packaged fruit juices consumption in patients with NAFLD as compared to that in control population. Other objectives included assessment of the independent role of sweetened soft drinks as a risk factor for NAFLD in the absence of metabolic syndrome and to correlate the level of steatosis (objectively graded by ultrasonography) with the amount of intake of soft drinks in patients of NAFLD.
\end{abstract}

Method: 50 patients of NAFLD as diagnosed clinically and by ultrasonography were identified and compared with 50 age matched control population reporting to the hospital with ailments other than NAFLD. The presence or absence of metabolic syndrome was noted in all the patients. The history of soft drink intake was obtained from the patient under following heads: a) quantity consumed, b) type of soft drink/ packaged juice consumed, c) period of ingestion and d) frequency of consumption.

Result: The average monthly consumption, duration of consumption and the mean total sugar consumption of sweetened soft drinks / packaged juices were significantly higher in patients with NAFLD versus those in the control group ( $\mathrm{p}=0.0002 ;<0.0001 ; \mathrm{p}=0.002$ respectively). The quantity and chronicity of intake correlated well with the grade of NAFLD. As the average monthly consumption and mean sugar consumption increased the grade of fatty liver on USG also increased $(\mathrm{p}=0.004)$. A total of 11 patients out of all the 100 patients (study and control groups combined) had a daily intake of $150-300 \mathrm{ml}$ of SSD for a period ranging from 5 to 20 years. Two of these had grade I NAFLD whereas 9 had grade II-III NAFLD. Five of these 11 patients had a daily intake of $250 \mathrm{ml}$ or greater. All these 5 patients had grade II-III NAFLD documented on ultrasound findings.

Conclusion: The consumption of sweetened soft drinks and packaged juice is on the rise in India. The intake of these drinks is significantly higher in patients with NAFLD.

Keywords: Nonalcoholic Fatty Liver Disease, Risk Factor, Soft Drinks, Metabolic Syndrome

\section{Introduction}

Nonalcoholic fatty liver disease (NAFLD) is characterized by hepatic steatosis, confirmed either by imaging or by histology and there are no causes for secondary hepatic fat accumulation such as significant alcohol consumption, use of steatogenic medication, viral diseases or hereditary disorders ${ }^{1}$. Normally, less than $5 \%$ of the liver is fat by weight, but in patients with nonalcoholic steatohepatitis (NASH), as much as $50 \%$ to $80 \%$ of liver weight may be made up of fat, mostly in the form of triglycerides (TG).

The dietary habits have drastically changed over last few decades. There has been a surge in introduction of sweeteners such as fructose and sucrose by the food industries. Regular aerated soft drinks and packaged fruit drinks have become major sources of high fructose or sugar $^{2}$. Consumption of sugar sweetened beverages per capita in India is reported to be about 11 litres a year, which is low compared with other nations as per 2013 report from the global marketing research firm Euromonitor International that also found that the consumption is increasing by $13 \%$ a year ${ }^{3}$. 1,80,000 deaths in the world in 2010 were reported due to consumption of sugar-sweetened beverages, with 72.3 per cent from diabetes, 24.2 per cent from cardiovascular disease and 3.5 per cent from cancers ${ }^{4}$.

NAFLD in majority of patients is associated with metabolic risk factors such as obesity, diabetes mellitus, and dyslipidemia ${ }^{1}$. Recent evidence suggests an association between the intake of sugar sweetened soft drinks and the risk of obesity and diabetes resulting from large amounts of fructose used in their manufacture, which raises blood glucose similar to sucrose ${ }^{5}$. The newer type of low calorie aerated soft drinks contain aspartame sweetener and 
caramel coloring, which are rich in advanced glycation end products that also increase insulin resistance and inflammation ${ }^{6}$. Research both in humans and animals has confirmed that diet rich in sucrose/fructose can affect fatty infiltration and lipid peroxidation in various types of liver disease including NAFLD ${ }^{6,8}$. The increased intake of soft drinks has been associated with NAFLD ${ }^{9}$. NAFLD patients have been detected to have 5 times more consumption of carbohydrates from soft drinks/packaged juice as compared to healthy persons ${ }^{10}$.

We studied/investigated this phenomenon in patients of NAFLD without features of metabolic syndrome with a hypothesis that excess soft drink intake can be an independent risk factor to cause non-alcoholic hepatic steatosis. The detailed objectives were: (i) To categorize NAFLD patients reporting to our gastroenterology clinic into two subgroups:- with /without metabolic syndrome; (ii) To determine the quantity, frequency, duration and type sweet soft drinks /packaged fruit juices consumption in these set of patients; (iii) Assess the association between consumption of soft drinks/packaged juices and fatty liver in patients with or without metabolic syndrome as against age-matched controls; (iv) To correlate the level of steatosis (objectively rated by USG) with the amount of intake of soft drinks in patients of NAFLD

\section{Materials and Methods}

Study Population: 50 patients of NAFLD diagnosed by clinical, radiological or histopathological criteria reporting to gastroenterology clinic were included in the study. The serum biochemical analysis, liver ultrasound and liver biopsy histopathology report were analyzed to confirm the diagnosis of NAFLD. 50 age-matched controls reporting to gastroenterology clinic with ailments other than NAFLD, which would not bias the findings, were also included in the study.

Data Collection: Following readings were obtained from the patient to confirm the presence of metabolic syndrome (NCEP2005 criteria): Waist hip ratio, Blood pressure values, Serum lipid profile and blood sugar levels ${ }^{51}$. The history of sweetened soft drink (SSD) intake was obtained from the patient under following heads: a) quantity consumed, b) type of soft drink/packaged juice consumed, c) period of ingestion and d) frequency of consumption. The fructose/ sugar content and calories $/ 100 \mathrm{ml}$ of commonly consumed soft drinks were deciphered from the market.

The NAFLD conventionally are graded and staged using 2005 NAS criteria on liver biopsy ${ }^{52}$. Herein the extent of steatosis, ballooning of hepatocytes, lobular inflammation and fibrosis is objectively used to objectively stratify the patient. The radiologist use a 4 grade system to grade the extent of fatty liver ${ }^{49,50}$. The sonographic grading criteria for NAFLD are ${ }^{50}$ : (i) Mild: Minimally increased parenchymal echogenicity and normal appearing intra hepatic vessel walls; (ii) Moderate: Further increased parenchymal echogenicity causing decreased resolution of intrahepatic vessel walls; (iii) Severe: Markedly increased parenchymal echogenicity causing inability to resolve intrahepatic vessel walls and obscured diaphragmatic outline.

Statistical Analysis: All the data were prospectively entered in excel sheets. Discrete categorical data were expressed as number and percentage. Continuous data were expressed as mean \pm standard deviation. Continuous variables were compared between the two groups using independent samples unpaired single tailed Student ' $t$ ' test. A p value $<0.05$ was considered as signifiant. Spearman correlation coefficient was used to correlate the objective radiological extent of NAFLD against the quantity of soft drink intake.

\section{Results}

The mean age of patients with NAFLD was 48.2 years with $72 \%$ of these cases showing presence of metabolic syndrome (Table 1).

The 50 patients of NAFLD were compared with 50 control patients. Both the groups were equally age and sex matched. The average monthly consumption, duration of consumption and the mean total sugar consumption of sweetened soft drinks / packaged juices were significantly higher in patients with NAFLD versus those in the control group (Table 2).

The radiological grade of NAFLD was also compared with the average monthly consumption, duration of consumption and the mean total sugar consumption of sweetened soft drinks / packaged juices. These were significantly higher in patients with grade II-III versus those in grade I patients (Table 3).

A total of 11 patients out of all the 100 patients (study and control groups combined) had a daily intake of $150-300 \mathrm{ml}$ of SSD for a period ranging from 5 to 20 years. Two of these had grade I NAFLD whereas 9 had grade II NAFLD. Five of these 11 patients had a daily intake of $250 \mathrm{ml}$ or greater. All these 5 patients had grade II-III NAFLD documented on ultrasound findings. Since only 2/50 cases had undergone a liver biopsy, no correlation of fat content in biopsy was done against the consumption of SD as it would have a been too small a number.

Patients with NAFLD were categorized into those with presence or absence of metabolic syndrome. There was no significant difference in the intake of sweetened soft drinks / packaged juices amongst these groups (Table 4). 
Patients with NAFLD but without the presence of metabolic syndrome were compared to the control group. The intake of sweetened soft drinks / packaged juices was significantly higher in this subgroup versus the control (Table 5). This finding indicates that sweetened soft drinks / packaged juices are independent risk factors for development of NAFLD in the absence of metabolic syndrome. The risk stratification increases in the presence of metabolic syndrome.

\section{Discussion}

Nonalcoholic fatty liver disease (NAFLD) is characterized by hepatic steatosis, with no secondary causes of steatosis:alcohol consumption, drugs, infections or hereditary

Table 1: Demographic details of patients with NAFLD $(\mathrm{N}=50)$.

\begin{tabular}{|l|l|}
\hline Age (Mean) & 48.2 years (range: 24-67 years) \\
\hline Sex (M/F) & $42 / 8$ \\
\hline Presence of Metabolic Syndrome & $36(72 \%)$ \\
\hline
\end{tabular}

Table 2: Consumption of Sweetened Soft Drinks / Packaged Juices.

\begin{tabular}{|c|c|c|c|}
\hline & NAFLD & Control & $p$ value \\
\hline $\mathrm{N}$ & 50 & 50 & \\
\hline Age (years) & $48.2 \pm 12$ & $45.1 \pm 12.6$ & 0.1 (NS) \\
\hline $\operatorname{Sex}(M / F)$ & $42 / 8$ & $43 / 7$ & \\
\hline Average Consumption (ml/month) & $1766 \pm 2789.6$ & $240 \pm 362.4$ & 0.0002 \\
\hline Period (years) & $14.84 \pm 11.6$ & $3.96 \pm 6.5$ & $<0.0001$ \\
\hline Mean Sugar consumption (gms) & $47492.66 \pm 82527.4$ & $2587.96 \pm 5478.6$ & 0.0002 \\
\hline
\end{tabular}

Table 3: Association of Grade of NAFLD with Intake of Sweetened Soft Drinks.

\begin{tabular}{|l|r|r|r|}
\hline & Grade I NAFLD & Grade II-III NAFLD & P value \\
\hline N & 37 & 13 & \\
\hline Average Consumption (ml/month) & $652.7 \pm 1212.9$ & $4934.61 \pm 3558.6$ & 0.0004 \\
\hline Period (years) & $13.43 \pm 11.4$ & $18.84 \pm 11.7$ & 0.08 \\
\hline Mean Sugar consumption (gms) & $25886.23 \pm 67062.3$ & $114112.5 \pm 92677.6$ & 0.004 \\
\hline
\end{tabular}

Table 4: Association of metabolic syndrome with Intake of Sweetened Soft Drinks in patients with NAFLD.

\begin{tabular}{|l|r|r|r|}
\hline & Metabolic Syndrome Present & Metabolic Syndrome absent & 14 \\
\hline N & 36 & $1271.43 \pm 2524.5$ & \\
\hline Average Consumption (ml/month) & $1958.33 \pm 2896.8$ & $12.14 \pm 11.4$ & 0.207 \\
\hline Period (years) & $15.88 \pm 11.6$ & $36613.92 \pm 69871.2$ & 0.15 \\
\hline Mean Sugar consumption (gms) & $51844.15 \pm 87637.5$ & 0.26 \\
\hline
\end{tabular}

Table 5: Association of Sweetened Soft Drinks / Packaged Juices with NAFLD in patients without metabolic syndrome.

\begin{tabular}{|c|c|c|c|}
\hline & NAFLD without Metabolic syndrome & Control & $p$ value \\
\hline $\mathrm{N}$ & 14 & 50 & \\
\hline Average Consumption (ml/month) & $1271.43 \pm 2524.5$ & $240 \pm 362.4$ & 0.07 \\
\hline Period (years) & $12.14 \pm 11.4$ & $3.96 \pm 6.5$ & 0.01 \\
\hline Total Consumption (ml) & $340714 \pm 672552.7$ & $20100 \pm 41934.1$ & 0.05 \\
\hline Mean Sugar consumption (gms) & $36613.92 \pm 69871.2$ & $2587.96 \pm 5478.6$ & 0.046 \\
\hline
\end{tabular}


disorders. It affects $2.8-24 \%$ of the general population ${ }^{11}$. The incidence of NAFLD in the Indian subcontinent ranges from $8-32 \%$ in different population settings (Table 1$)^{12-17}$. The natural history of the disease is still unclear. NAFLD can progress to nonalcoholic steatohepatitis (NASH), a fatty liver with hepatitis. This form of liver injury carries a $20 \%$ $50 \%$ risk for progressive fibrosis, $30 \%$ risk for cirrhosis, and $5 \%$ risk for hepatocellular carcinoma $(\mathrm{HCC})^{18-20}$. In studies with paired biopsies (one biopsy performed after the period of follow-up), the disease progressed in $32-41 \%$, remained stable in $34-50 \%$, and improved in a minority of the patients with NAFLD.

The dietary habits have drastically changed over last few decades. There has been a surge in introduction of sweeteners such as fructose and sucrose by the food industries. Regular aerated soft drinks and packaged fruit drinks have become major sources of high fructose or sugar. It is well known that dietary factors can affect fatty infiltration and lipid peroxidation in various types of liver disease including NAFLD ${ }^{23,24}$. Increased ingestion of SD has been found to be one of the dietary factors linked to NAFLD independent of metabolic syndrome.

The term SSD (soda, soda pop, pop, colas etc) refers to a nonalcoholic beverage that is usually carbonated. Two types of SSD are used; regular SSD which are sweetened with sugar (fructose) and diet SSD which are sweetened with non-caloric sweeteners (aspartame). High fructose corn syrup (HFCS) is used almost exclusively as a sweetener because of its lower cost. HFCS made by enzymatic isomerization of glucose to fructose was introduced as HFCS-42 (42\% fructose) and HFCS-55 (55\% fructose) in 1967 and 1977, respectively. Worldwide, SD is the leading cause of added sugar. Recent evidence suggests an association between the intake of sugar sweetened SD and the risk of obesity and diabetes resulting from large amounts of high fructose corn syrup (HFCS) used in their manufacture, which raises blood glucose similar to sucrose $^{5}$. In addition, diet SD contain aspartame sweetener and caramel coloring, which are rich in advanced glycation end products that potentially increase insulin resistance and inflammation ${ }^{6,26}$. Increased ingestion of SD has been found to be linked to NAFLD independent of metabolic syndrome. Assy et al proved that increased ingestion of soft drinks was found to be linked to NAFLD independent of metabolic syndrome ${ }^{9}$. It is conjectured that hepatic fructose metabolism begins with phosphorylation of fructokinase. Fructose carbon enters the glycolyticpathway at the triose phosphate level. Fructose thereby bypasses the main regulator point by which glucose carbon enters glycolysis. This permits excess fructose to function as an unregulated source of glycerol-3-phosphate and acetyl-CoA for hepatic lipogenesis ${ }^{10}$.

In our study, the intake of sweetened Soft Drinks (SSD) I packaged juices was found to be statistically higher in patients with NAFLD versus that in controls (1766 $\mathrm{ml} /$ month vs $240 \mathrm{ml} / \mathrm{month}$; $\mathrm{p}<0.0002$ ) (Table 2). The chronicity of intake also mattered and was significantly higher in the NAFLD group $(\mathrm{p}<0.0001)$. We graded the degree of NAFLD based on ultrasound criteria and grouped the patients into 2 categories: Low grade (grade I) and High Grade (II-III). The intake of SSDs was again significantly higher in high grade NAFLD as compared to low grade NAFLD $(p=0.0004)$ indicating that SSDs are not just linked to the higher incidence of NAFLD but also to the grade of NAFLD (Table 3). A total of 11 patients out of all the 100 patients (study and control groups combined) had a daily intake of $150-300 \mathrm{ml}$ of SSD for a period ranging from 5 to 20 years. Two of these had grade I NAFLD whereas 9 had grade II NAFLD. Five of these 11 patients had a daily intake of $250 \mathrm{ml}$ or greater. All these 5 patients had grade IIIII NAFLD documented on ultrasound findings indicating the risk of NAFLD amongst people with daily high volume intake of SSDs. Our results correlate well with those of Assy et al who found a significant correlation between the amount of soft drink consumed and the extent of fatty infiltration ${ }^{25}$.

The amount of sugar consumed due to sweetened Soft Drinks (SSD) / Packaged Juices was found to be statistically higher in patients with NAFLD versus that in controls in our study ( $<<0.0002)$. In a study by Abid et al, the NAFLD group consumed five times more carbohydrates from soft drinks compared to healthy controls ${ }^{26}$.

We categorized patients with NAFLD into those with and those without metabolic syndrome (Table 4). The intake of SSD did not differ significantly amongst both the groups. This probably hints towards SSDs being an independent risk factor in the etiology of NAFLD. Patients with NAFLD and without metabolic syndrome were compared with controls (Table 5). The intake of SSDs was again significantly higher in these patients as compared to the controls $(\mathrm{p}=0.05)$. This proves that SSDs are an independent risk factor in the development of NAFLD. Studies by Assy et $\mathrm{al}^{25}$ and Siddiqi et $\mathrm{al}^{47}$ also documented similar results. Assy et al studied the association between soft drink consumption and the presence of fatty liver in NAFLD patients who do not have classic risk factors. They observed that $80 \%$ of patients ( 25 of 31 ) consumed an excessive amount of soft drink beverages (more than 50 g/day of added sugar) for 36 months, compared with $20 \%$ in healthy controls. 
Recent evidences regarding the association of fructose and soft drinks with NAFLD however have been conflicting. Armiliato and colleagues and a recent meta-analysis did a review of existing literature and found that even though growing evidence suggests that fructose contributes to the development and severity of NAFLD, available evidence is not sufficiently robust to draw conclusions regarding effects of fructose, HFCS, or sucrose consumption on NAFLD $^{43-45}$. Further studies would be needed to highlight the individual role of each of these in the development of NAFLD and other diseases like diabetes.

\section{Conclusion}

The consumption of sweetened soft drinks and packaged juice is on the rise in India. The intake of these drinks is significantly higher in patients with NAFLD. Increasing consumption of these drinks is an independent risk factor not only for the development of NAFLD but also for a higher grade of NAFLD especially in population with high daily and chronic intake. It is important to highlight the hazardous effects of regular ingestion of these drinks to the population.

Declarations: The study was funded through ICMR as a STS project in 2017-18

\section{References}

1. Chalasani N, Younossi Z, Lavine JE, Diehl AM, Brunt EM, Cusi K et al. American Gastroenterological Association.; American Association for the Study of Liver Diseases.; American College of Gastroenterology. The diagnosis and management of non-alcoholic fatty liver disease: practice guideline by the American Gastroenterological Association, American Association for the Study of Liver Diseases, and American College of Gastroenterology. Gastroenterology. 2012 Jun; 142(7):1592-609.

2. Boulton J, Hashem KM, Jenner KH, Lloyd-Williams F, Bromley H, Capewell S. How much sugar is hidden in drinks marketed to children? A survey of fruit juices, juice drinks and smoothies. BMJ Open. 2016 Mar 23; 6(3):e010330.

3. Bhaumik $\mathrm{S}$. The public health threat from sugary drinks in India. BMJ. 2014 Oct 21; 349: g6216.

4. Singh GM, Micha R, Khatibzadeh S, Lim S, Ezzati M, on behalf of the Global Burden of Diseases Nutrition and Chronic Diseases Expert Group (NutriCoDE) D. Estimated Global, Regional, and National Disease Burdens Related to Sugar-Sweetened Beverage Consumption in 2010. Circulation. 2015;132 (8):639-666.

5. Gaby AR. Adverse effects of dietary fructose. Altern Med Rev. 2005;10:294-306.

6. Vlassara H, Cai W, Crandall J, Goldberg T, Oberstein R, Dardaine V, Peppa M, Rayfield EJ. Inflammatory mediators are induced by dietary glycotoxins, a major risk factor for diabetic angiopathy. Proc Natl Acad Sci USA. 2002;99:15596-15601.

7. Mezey E. Dietary fat and alcoholic liver disease. Hepatology. 1998;28:901-905.

8. Fernández MI, Torres MI, Gil A, Ríos A. Steatosis and collagen content in experimental liver cirrhosis are affected by dietary monounsaturated and polyunsaturated fatty acids. Scand J Gastroenterol. 1997;32:350-356.

9. Assy N, Nasser G, Kamayse I, Nseir W, Beniashvili Z, Djibre A, Grosovski M. Soft drink consumption linked with fatty liver in the absence of traditional risk factors. Can J Gastroenterol. 2008;22:811-816.

10. Abid A, Taha O, Nseir W, Farah R, Grosovski M, Assy N. Soft drink consumption is associated with fatty liver disease independent of metabolic syndrome. J Hepatol. 2009;51:918-924.

11. Huang PL. A comprehensive definition for metabolic syndrome. Dis Model Mech. 2009 May-Jun; 2(5-6): 231237

12. Kleiner DE, Brunt EM, et al. Design and validation of a histological scoring system for nonalcoholic fatty liver disease. Hepatology 2005;41:1313-21.

13. Dasarathy S, Dasarathy J, Khiyami A, et al. Validity of real time ultrasound in the diagnosis of hepatic steatosis: A prospective study. J Hepatol 2009; 51:1061- 7.

14. Riley TR, Bruno MA. Sonographic measurement of the thickness of subcutaneous tissues in nonalcoholic fatty liver disease versus other chronic liver diseases. J Clin Ultrasound. 2005; 33:439-441.

15. Singh D, Das CJ, Baruah MP. Imaging of non alcoholic fatty liver disease. A road less travelled. Indian J Endocr Metab 2013;17:990-995

16. Browning JD, Szczepaniak LS, Dobbins R, Nuremberg P, Horton JD, Cohen JC, Grundy SM, Hobbs HH. Prevalence of hepatic steatosis in an urban population in the United States: impact of ethnicity. Hepatology. 2004;40:1387-1395.

17. Majumdar A, Misra P, Sharma S, Kant S, Krishnan A, Pandav CS. Prevalence of nonalcoholic fatty liver disease in an adult population in a rural community of Haryana, India. Indian J Public Health 2016;60:26-33.

18. Mohan V, Farooq S, Deepa M, Ravikumar R, Pitchumoni CS. Prevalence of non-alcoholic fatty liver disease in urban South Indians in relation to different grades of glucose intolerance and metabolic syndrome. Diabetes Res Clin Pract 2009;84:84-91.

19. Amarapurkar D, Kamani P, Patel N, Gupte P, Kumar P, Agal $\mathrm{S}$, et al. Prevalence of non-alcoholic fatty liver disease: Population based study. Ann Hepatol 2007;6:161-3.

20. Das K, Das K, Mukherjee PS, Ghosh A, Ghosh S, Mridha $\mathrm{AR}$, et al. Nonobese population in a developing country has a high prevalence of nonalcoholic fatty liver and significant liver disease. Hepatology 2010;51:1593-602. 
21. Singh SP, Nayak S, Swain M, Rout N, Mallik RN, Agrawal $\mathrm{O}$, et al. Prevalence of non-alcoholic fatty liver disease in coastal eastern India: A preliminary ultrasonographic survey. Trop Gastroenterol 2004;25:76-9.

22. Bajaj S, Nigam A, Luthra A, Pandey RM, Kondal D, Bhatt SP, et al. A case-control study on insulin resistance, metabolic co-variates \& prediction score in non-alcoholic fatty liver disease. Indian J Med Res 2009;129:285-92.

23. Bugianesi E, Leone N, Vanni E, Marchesini G, Brunello F, Carucci P, Musso A, De Paolis P, Capussotti L, Salizzoni $\mathrm{M}$, et al. Expanding the natural history of nonalcoholic steatohepatitis: from cryptogenic cirrhosis to hepatocellular carcinoma. Gastroenterology. 2002;123:134-140.

24. Willner IR, Waters B, Patil SR, Reuben A, Morelli J, Riely CA. Ninety patients with nonalcoholic steatohepatitis: insulin resistance, familial tendency, and severity of disease. Am J Gastroenterol. 2001;96:2957-2961.

25. Angulo P. Nonalcoholic fatty liver disease. N Engl J Med. 2002;346:1221-1231.
26. Hofmann SM, Dong HJ, Li Z, Cai W, Altomonte J, Thung SN, Zeng F, Fisher EA, Vlassara H. Improved insulin sensitivity is associated with restricted intake of dietary glycoxidation products in the db/db mouse. Diabetes. 2002;51:2082-2089.

27. Siddiqi Z, Karoli R, et al. Soft Drinks Consumption and the Risk of Nonalcoholic Fatty Liver Disease. JAPI 2017;65:28-32.

28. Armiliato et al. High-fructose Intake in Obesity-related Nonalcoholic Fatty Liver Disease. J Gastrointest Dig Syst 2015, 5:3.

29. Kanerva N, Sandboge S, Kaartinen NE, Männistö S, Eriksson JG (2014) Higher fructose intake is inversely associated with risk of nonalcoholic fatty liver disease in older Finnish adults. Am J Clin Nutr 100: 1133-1138.

30. Chung M, Ma J, Patel K, Berger S, Lau J, et al. (2014) Fructose, highfructose corn syrup, sucrose, and nonalcoholic fatty liver disease or indexes of liver health: a systematic review and meta-analysis. Am J Clin Nutr 100: 833-849.

*Corresponding author:

Gp Capt Prateek Kinra, Postal address: Prof \& Offg HoD,Dept. Of Pathology, Armed Forces Medical College, Wanowrie, Pune- 411040 INDIA

Phone: +91 9945277110

Email: pkinra_in@yahoo.com

Date of Submission : 05/01/2020

Financial or other Competing Interests: None. 